\title{
Towards understanding androgen receptor-independent prostate cancer: an evolving paradigm
}

\author{
Jianfu Zhou ${ }^{1,2,3}$, Yuliang Wang ${ }^{3}$, Songtao Xiang ${ }^{1}$, Franky L. Chan ${ }^{3}$ \\ ${ }^{1}$ Department of Urology, The Second Affiliated Hospital of Guangzhou University of Chinese Medicine, Guangzhou 510370, China; ${ }^{2}$ The Second \\ Clinical College, Guangzhou University of Chinese Medicine, Guangzhou 510006, China; ${ }^{3}$ School of Biomedical Sciences, Faculty of Medicine, The \\ Chinese University of Hong Kong, Hong Kong, China \\ Correspondence to: Franky L. Chan. School of Biomedical Sciences, The Chinese University of Hong Kong, Shatin, Hong Kong, China. \\ Email: franky-chan@cuhk.edu.hk. \\ Comment on: Liu Y, Horn JL, Banda K, et al. The androgen receptor regulates a druggable translational regulon in advanced prostate cancer. Sci \\ Transl Med 2019. doi: 10.1126/scitranslmed.aaw4993.
}

Submitted Dec 12, 2019. Accepted for publication Dec 26, 2019.

doi: $10.21037 /$ tcr.2020.01.25

View this article at: http://dx.doi.org/10.21037/tcr.2020.01.25

Prostate cancer (PC) arises as a distinctive androgen-driven malignancy, therefore androgen-deprivation therapy (ADT) targeting androgen receptor (AR) represents the mainstay treatment for conventional advanced adenocarcinoma. Although initially effective, a majority of tumors relapse with progression to a lethal castration-resistant prostate cancer (CRPC) (1). The latest use of next-generation AR signaling inhibitors (ARSIs), such as the steroidogenic enzyme CYP17A1 inhibitor abiraterone and antiandrogen enzalutamide, have resulted in life-extending benefits for the management of CRPC $(2,3)$. However, a subset of highly aggressive transdifferentiated tumors emerges as AR-null/deficient/low heterogeneous phenotype with or without neuroendocrine prostate cancer (NEPC), which is characterized by the phenotypic shift of epithelial plasticity to a histologic subtype that morphologically resembles small-cell malignancy, resulting in eventual resistance to AR-directed therapies (4). The prognosis of these subtype of tumors is extremely poor and few treatment options exist.

The AR-independent plasticity is an emerging clinical entity in PC heterogeneity, especially in the nextgeneration $\mathrm{AR}$ antagonism era. In the era before the FDA approval of abiraterone and enzalutamide (1997-2011), most CRPCs presented AR-positive prostate cancer (ARPC; $85 \%$ ) with few NEPCs (10\%) and fewer AR-/ NE- tumors (5\%), thereby termed as "double-negative" PCs (DNPC). Whereas, in the current era with the clinical introduction of next-generation ARSIs (2012-2016), a shift of elevated AR-/NE- DNPC tumors (21\%) and unaltered NEPCs (10\%) were observed in a small minority of patients with both ARPC and NEPC tumors (5). A multiinstitutional prospective study reported a $17 \%$ detection of overall incidence for treatment-emergent small-cell neuroendocrine prostate cancer ( $\mathrm{t}-\mathrm{SCNC}$ ) of in a total of 202 mCRPC patients undergoing metastatic tumor biopsy. Most tumors (75\%) showed upregulated nuclear AR protein expression and high serum PSA ( $>60 \mathrm{ng} / \mathrm{mL})$, despite the classical features with AR-null phenotype and low serum PSA levels for de novo SCNC (6). Similarly, results from a recent study by Abida $e t$ al. showed that prostate tumors collected from 128 mCRPC patients treated with first-line next-generation ARSIs presented enriched histopathologic NE features (10.5\%) after exposure to ARSIs as compared to ARSIs-naive tumors (2.3\%) (7). These studies suggest that the heterogeneous process is an important mechanism responsible for the development of treatment-resistant mCRPC.

As to the underlying molecular determinants and mediators responsible for the heterogeneous setting, Liu and colleagues recently observed that a deficiency in AR abundance could lead to an increase of eIF4F-regulated translation initiation or protein synthesis in PTEN-deficient PC, which indicating a link between translational regulon and AR plasticity (8). Previous studies identified a few lineage plasticity-associated molecular events, for example genomic loss of the tumor suppressors RB1 and TP53 (9), 
upregulation of lineage pluripotency transcription factors such as SOX2 (10), N-MYC (11) and ONECUT2 (12), epigenetic modifications including upregulation of EZH2 (13), elevation of FGF and MAPK kinase activities (5), dysregulation of Notch receptor pathway inhibitory ligand DLL3 (14), and overexpression of TMPRSS2$E R G$ fusion gene (15), etc. In addition, we also recently demonstrated that an orphan nuclear receptor TLX is capable of transcriptional repression of AR expression via an epigenetic mechanism, which contributes to AR plasticity and androgen insensitivity in CRPC (16).

Understanding the process and development of ARindependent PC can own certain translational relevance to clinical practice. Although the loss of AR expression eliminates the AR signaling as a therapeutic target, acquired treatment-emergent features of NEPC and other cancer stem-like cell phenotypes can exhibit novel targets and vulnerabilities. For instance, work led by Liu and colleagues revealed that $\mathrm{PC}$ patients presenting an upregulated translation initiation particularly with AR-low setting, may get benefit from the emerging eIF4F-targeted therapeutics. Indeed, the protein synthesis inhibitors are currently under investigation of phase- 1 and -2 clinical trials (8). Other available drugs bypassing AR antagonism and designed with rationale of combination or co-targeting strategies, such as targeting AURK/RB1 axis, EZH2, DLL3, and FGF/ MAPK, are also in development for AR-null/deficient/low or NEPC tumors (17).

In conclusion, lineage plasticity associated with loss of AR signaling dependence and the acquisition of $\mathrm{NE}$ features occurs in an approximately $15 \%$ of advanced PC patients. Understanding the district phenotypic shifts not only helps to gain insights into mechanisms underlying therapyresistance but also provides novel strategies bypassing AR antagonism. Future studies are proposed to further outline the plasticity landscape in order to move forward our knowledge in treating transdifferentiated subtype tumors.

\section{Acknowledgments}

Funding: Jianfu Zhou was awarded by the Eternal Flame Scholar Programme of Guangzhou University of Chinese Medicine. This work was in part supported by the Guangdong-Hong Kong collaborative innovation projects from Guangdong Provincial Hospital of Chinese Medicine (grant YN2018HK02) and Department of Science and Technology of Guangdong Province (grant
2017A050506042).

\section{Footnote}

Provenance and Peer Review: This article was commissioned and reviewed by the Section Editor Dr. Peng Zhang (Department of Urology, Zhongnan Hospital of Wuhan University, Wuhan, China).

Conflicts of Interest: The authors have completed the ICMJE uniform disclosure form (available at http://dx.doi. org/10.21037/tcr.2020.01.25). The authors have no conflicts of interest to declare.

Ethical Statement: The authors are accountable for all aspects of the work in ensuring that questions related to the accuracy or integrity of any part of the work are appropriately investigated and resolved.

Open Access Statement: This is an Open Access article distributed in accordance with the Creative Commons Attribution-NonCommercial-NoDerivs 4.0 International License (CC BY-NC-ND 4.0), which permits the noncommercial replication and distribution of the article with the strict proviso that no changes or edits are made and the original work is properly cited (including links to both the formal publication through the relevant DOI and the license). See: https://creativecommons.org/licenses/by-nc$\mathrm{nd} / 4.0 /$.

\section{References}

1. Karantanos T, Evans CP, Tombal B, et al. Understanding the mechanisms of androgen deprivation resistance in prostate cancer at the molecular level. Eur Urol 2015;67:470-9.

2. de Bono JS, Logothetis CJ, Molina A, et al. Abiraterone and increased survival in metastatic prostate cancer. $\mathrm{N}$ Engl J Med 2011;364:1995-2005.

3. Tran C, Ouk S, Clegg NJ, et al. Development of a secondgeneration antiandrogen for treatment of advanced prostate cancer. Science 2009;324:787-90.

4. Davies AH, Beltran H, Zoubeidi A. Cellular plasticity and the neuroendocrine phenotype in prostate cancer. Nat Rev Urol 2018;15:271-86.

5. Bluemn EG, Coleman IM, Lucas JM, et al. Androgen receptor pathway-independent prostate cancer is sustained 
through FGF signaling. Cancer Cell 2017;32:474-89. e6.

6. Aggarwal R, Huang J, Alumkal JJ, et al. Clinical and genomic characterization of treatment-emergent smallcell neuroendocrine prostate cancer: A multi-institutional prospective study. J Clin Oncol 2018;36:2492-503.

7. Abida W, Cyrta J, Heller G, et al. Genomic correlates of clinical outcome in advanced prostate cancer. Proc Natl Acad Sci U S A 2019;116:11428-36.

8. Liu Y, Horn JL, Banda K, et al. The androgen receptor regulates a druggable translational regulon in advanced prostate cancer. Sci Transl Med 2019. doi: 10.1126/ scitranslmed.aaw4993.

9. Ku SY, Rosario S, Wang Y, et al. Rb1 and Trp53 cooperate to suppress prostate cancer lineage plasticity, metastasis, and antiandrogen resistance. Science 2017;355:78-83.

10. Mu P, Zhang Z, Benelli M, et al. SOX2 promotes lineage plasticity and antiandrogen resistance in TP53- and RB1deficient prostate cancer. Science 2017;355:84-8.

11. Berger A, Brady NJ, Bareja R, et al. N-Myc-mediated epigenetic reprogramming drives lineage plasticity in advanced prostate cancer. J Clin Invest 2019;130:3924-40.

12. Guo H, Ci X, Ahmed M, et al. ONECUT2 is a driver

Cite this article as: Zhou J, Wang Y, Xiang S, Chan FL. Towards understanding androgen receptor-independent prostate cancer: an evolving paradigm. Transl Cancer Res 2020;9(2):415-417. doi: 10.21037/tcr.2020.01.25 of neuroendocrine prostate cancer. Nat Commun 2019;10:278.

13. Dardenne E, Beltran H, Benelli M, et al. N-Myc Induces an EZH2-Mediated Transcriptional Program Driving Neuroendocrine Prostate Cancer. Cancer Cell 2016;30:563-77.

14. Puca L, Gavyert K, Sailer V, et al. Delta-like protein 3 expression and therapeutic targeting in neuroendocrine prostate cancer. Sci Transl Med 2019. doi: 10.1126/ scitranslmed.aav0891.

15. Blee AM, He Y, Yang Y, et al. TMPRSS2-ERG Controls Luminal Epithelial Lineage and Antiandrogen Sensitivity in PTEN and TP53-Mutated Prostate Cancer. Clin Cancer Res 2018;24:4551-65.

16. Jia L, Wu D, Wang Y, et al. Orphan nuclear receptor TLX contributes to androgen insensitivity in castration-resistant prostate cancer via its repression of androgen receptor transcription. Oncogene 2018;37:3340-55.

17. Beltran H, Hruszkewycz A, Scher HI, et al. The Role of Lineage Plasticity in Prostate Cancer Therapy Resistance. Clin Cancer Res 2019;25:6916-24. 